\title{
AUTOESTIMA EN JÓVENES INDÍGENAS: BORUCAS Y TÉRRABAS ${ }^{1}$
}

\section{SELF-ESTEEM IN YOUNG INDIGENOUS: BORUCAS AND TERRABAS}

\author{
Marianela Román Solano* \\ Marjorie Moreno Salas**
}

\begin{abstract}
RESUMEN
En el presente documento se dan a conocer los resultados de un estudio que se basó en la exploración de la autoestima y autoconcepto en jóvenes de dos etnias indígenas (los Térrabas y los Borucas). Participaron 170 personas: 72 Térrabas y 98 Borucas. En ambos casos se denotan resultados satisfactorios con respecto a la identidad personal de los y las jóvenes participantes.
\end{abstract}

PALABRAS CLAVE: COSTA RICA * AUTOESTIMA * POBLACIÓN INDÍGENA * BORUCAS * TÉRRABAS * JUVENTUD

\section{ABSTRACT}

The present paper gives the results of a study that was based on exploration in selfesteem in two ethnic groups (Térrabas and Borucas). In this, 170 young people participated: 72 Térrabas and 98 Borucas. Both cases show good results with respect to the personal identity of young participants.

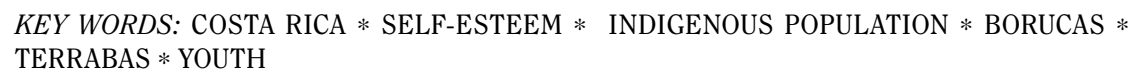

Este artículo corresponde a uno de los objetivos del Trabajo Final de Graduación de Marianela Román, autora principal, quien estudió el tema: "Relación entre las estrategias de aculturación y la identidad étnica y personal de adolescentes indígenas borucas". Ambas autoras agradecen al personal del Instituto de Investigaciones Psicológicas por la posibilidad de inserción de este trabajo dentro del proyecto "Percepción de discriminación y estrategias de afrontamiento". Además un especial agradecimiento a la Doctora Vanessa Smith coordinadora del proyecto.

* $\quad$ Asociación Nacional de Educadores (ANDE), Costa Rica: Encargada de la Oficina de Psicología. maroso4@hotmail.com

** Escuela de Psicología, Universidad de Costa Rica y Escuela de Psicología, Universidad Católica de Costa Rica. maryyuri12@gmail.com 


\section{INTRODUCCIÓN}

La autoestima es uno de los elementos que se considera clave para determinar el bienestar psicológico, es por tanto, un indicador del grado de satisfacción que una persona tiene consigo misma, y está a su vez fuertemente determinada por las condiciones de existencia.

Los grupos indígenas en Costa Rica constituyen una minoría étnica caracterizada históricamente por ser objeto de discriminación $y$ prejuicio (Bozzoli y Guevara, 2002), al vivir una exclusión estructural principalmente en el acceso a educación, trabajo, vivienda y además reflejada en actitudes $y$ prácticas de rechazo por sus condiciones de vida y cultura.

El significado subjetivo de pertenecer a un grupo étnico es históricamente reconocido como un componente central de la definición del sí mismo, especialmente como lo señala Smith, para aquellos que son víctimas de la discriminación racial y étnica, la relación cognitiva y emocional con el grupo étnico de referencia es aún más relevante para la evaluación y valoración personal (2002).

De esta forma, llama la atención identificar cómo están los jóvenes indígenas concibiendo $y$ valorando su persona, debido a que algunos estudios reflejan que las experiencias de discriminación étnica tienen un impacto negativo en la autoestima, pero a la vez se asume que frente a la discriminación, las personas tienden a presentar una identificación con el propio grupo, lo que a su vez tiene un efecto positivo en la autoestima (Smith, 2005).

Es así como el interés de este artículo radica en la exploración de la percepción y valoración del sí mismo en jóvenes indígenas, quienes atraviesan una etapa de la vida medular para la construcción de la identidad. Los grupos indígenas participantes están ubicados en el sur del país, a 300 kilómetros al sureste de San Isidro del General y son comunidades caracterizadas por un proceso de aculturación importante, que fue acentuado con la construcción de la carretera Interamericana (1936-1960), la cual vino a modificar el estilo de vida de las poblaciones de la zona.

La comunidad de Boruca está ubicada al sureste de Costa Rica, hacia la costa pacífica, y es el distrito 4 del cantón de Buenos Aires, en la provincia de Puntarenas. Para visitar la comunidad de Boruca, existen dos caminos: la ruta Térraba y Chánguina son unos $20 \mathrm{~km}$ de lastre $y$ barro, $y$ el otro camino es el que conduce a la carretera Interamericana, de solo $8 \mathrm{~km}$ de distancia.

Según el censo del 2000 para ese año la comunidad Boruca la habitaban 1386 personas consideradas indígenas, lo que equivale a un $46 \%$ del total de la población de la comunidad, los borucas además representan alrededor del $15 \%$ de las personas indígenas del país (Bozzoli y Guevara, 2002).

La principal actividad económica de la zona es la agricultura y otra fuente de ingresos es la artesanía, especialmente las máscaras y los productos textiles.

En el plano cultural se conserva la Fiesta de los Diablitos, que celebran los Borucas el 30 de diciembre, esta consiste en una representación de la lucha entre españoles e indígenas y es una actividad de mucho atractivo para la población joven de la comunidad.

Por su parte, la comunidad de Térraba está ubicada $16 \mathrm{~km}$ al sureste del puente sobre el río General en Buenos Aires, provincia de Puntarenas, sobre la carretera Interamericana $y$ de ahí $5 \mathrm{~km}$ al noroeste. Los Térrabas hablan español, son un grupo hermano de los Teribes panameños, y representan el $2 \%$ del total de la población indígena del país (Bozzoli y Guevara, 2002).

En la actualidad los Térrabas costarricenses viven una situación difícil pues conforman un grupo muy reducido, de los 1500 habitantes de la reserva, sólo el $40 \%$ son indígenas, incluso, sólo el $25 \%$ de las tierras está en sus manos (Funcoopa y Jetsay, 1999). Perdieron su lengua indígena desde hace muchos años, pero no han perdido su identidad cultural. Su principal actividad económica es también la agricultura y algunas actividades artesanales.

En lo referente a servicios, ambas comunidades cuentan con el acceso básico a servicios de salud $y$ alimentación $y$ tienen características similares a las de un pueblo rural costarricense, con una iglesia católica en el centro del poblado, una Escuela, un Colegio, Centro de Salud EBAIS, almacenes o pulperías, cantinas y las 
casas de habitación dispersas, cabe destacar que en ambos pueblos se cuenta con instituciones de educación secundaria, existen los Liceos de Térraba y Boruca.

A continuación, se presentan los antecedentes del estudio en cuestión, para ello se inicia una revisión de estudios internacionales hasta aproximarse a los nacionales.

Un estudio realizado por Delgado (2007), indagó las implicaciones del compromiso étnico sobre el bienestar psicológico y la identidad étnica indígena, dentro de los indicadores de bienestar se encontró la exploración de la autoestima. El estudio fue realizado con jóvenes procedentes de distintas tribus indígenas de los Estados Unidos, el contexto de investigación se dio en Haskell University, único centro de educación superior inter-tribal en los Estados Unidos.

Las hipótesis del estudio fueron: que la auto-representación de los jóvenes a través de categorías tribales como ser apache o comanche se va asociar con indicadores de bienestar personal y la segunda que a mayor grado de identificación y compromiso con el grupo étnico, mayor bienestar personal.

En el estudio participaron 188 jóvenes, de ellos 89 eran hombres y 98 mujeres, con edades entre 18 y 24 años, además 47 mujeres y 51 hombres no habían vivido nunca en reservas indígenas, mientras que 51 mujeres y 36 hombres si habían tenido esa experiencia, (en los datos anteriores solo se reportan los valores válidos, pues algunos informantes no contestaron las preguntas descritas).

Dentro de los resultados se reflejaron buenas puntuaciones en la autoestima, sin embargo, las mujeres que se identifican con sus grupos indígenas específicos tienen puntuaciones más bajas en la autoestima. Por otro lado, se constató una fuerte relación entre la identidad étnica y la autoestima, también se constató que el compromiso con las prácticas étnicas en jóvenes que provenían de reservas, estaba asociado con sentimientos de alta autoestima.

Por su parte, las jóvenes que no tenían experiencia de vivir en reservas pero que sí se definían como pertenecientes a grupos tribales reportaron alta satisfacción con su apariencia física y con las impresiones que ellas causaban en los otros. Se reflejó además que dicha experiencia se vincula con un mayor sentido de pertenencia, lo que puede a su vez estar ligado con una mayor autoestima personal y con buenos resultados en las habilidades académicas que los jóvenes que no han vivido en reservas.

Ahora bien, es importante hacer una revisión de lo estudiado en el país con respecto a la autoestima y las relaciones interétnicas. Hasta el momento no se ha registrado un estudio que explore el tema de la valía y conceptualización personal en indígenas costarricenses, pero la temática de la autoestima ha sido abordada por Smith en otros grupos étnicos, en este apartado se hace referencia a algunos de ellos.

Smith $(2002 a)$ realizó una revisión de la literatura internacional sobre identidad étnica de la población infantil pertenecientes a grupos étnicos minoritarios, $y$ encontró que la formación de la identidad étnica es un proceso multidimensional y dinámico, en donde la integración de la etnicidad en las percepciones del sí mismo depende de: 1. Aspectos socioculturales como el estatus social, la estructura, el tamaño y la organización de los grupos étnicos particulares, así como de las relaciones interétnicas a lo interno de las sociedades. 2 . El contexto inmediato, como la familia, la escuela, la zona de residencia $y$ los grupos de pares $y$; 3 . Los factores interindividuales ligados a la identidad personal que incluyen el desarrollo particular de capacidades cognitivas $y$ emocionales, las inclinaciones $y$ gustos personales y la autoestima.

En otro estudio de la misma autora (2002b) se evalúa un modelo teórico sobre el efecto de la discriminación percibida, el contacto interétnico, la identificación étnica y las actitudes interétnicas en la autoestima de 407 jóvenes de la minoría étnica afrocostarricense y 768 jóvenes del grupo mayoritario costarricense (blancos o mestizos), los resultados indicaron que la autoestima se ve directamente afectada por la identificación étnica y las actitudes interétnicas en ambos grupos culturales. Estas a su vez, están directamente influenciadas por la percepción de la discriminación y el contacto interétnico.

Así mismo, Smith (2002b) señaló que la vinculación cognitiva $y$ afectiva con los grupos étnicos (y principalmente con el endogrupo) 
tiene un relevante impacto en la definición y valoración del sí mismo tanto en miembros de grupos étnicos minoritarios como en miembros de grupos étnicos mayoritarios.

Según Smith, tanto la Teoría de la Identidad Social como los modelos bidimensionales de aculturación muestran que los grupos socioculturales tienen un importante impacto en la definición del sí mismo. La investigación muestra que una vinculación positiva con el grupo étnico de referencia representa una base más sólida para la autoestima que el abandonar los lazos con los grupos de referencia.

En un tercer estudio de Smith (2002b), se examinó el impacto relativo de cuatro variables en las actitudes interétnicas de 766 jóvenes "mestizo/blancos" de Limón y San José, una de ellas fue la autoestima personal y colectiva. Los resultados revelaron que la relación entre la autoestima (personal y colectiva) y las actitudes interétnicas varían de acuerdo con la zona de residencia, el género y el estatus socioeconómico de las personas participantes.

La investigación reflejó que la valoración del sí mismo y las actitudes inter-étnicas están vinculadas de manera positiva, apoyando la hipótesis de que la autoestima baja repercute en actitudes menos favorables hacia otros grupos, pero sólo en el caso de los hombres, los jóvenes de Limón, y los jóvenes de estratos socioeconómicos bajos. Esto indicaría según la autora que la diferenciación intergrupal, en efecto sirve como estrategia para lidiar con una autoestima personal baja, pero sólo para algunas personas y bajo ciertas circunstancias.

Para identificar las dos dimensiones exploradas en la presente investigación se va a entender autoestima según la definición de Engler (1998):

El conjunto de imágenes, pensamientos $y$ sentimientos que el individuo tiene de sí mismo. Pensamientos y sentimientos que, consecuentemente permiten diferenciar dos componentes o dimensiones de los mismos: los cognitivos y los evaluativos. (p.330).

De esta forma se concibe la autoestima desde dos componentes determinantes: la autoimagen o auto-concepto y la autovaloración.

La autoimagen representa una estructura cognitiva que se deriva de la interacción, ya que, por ejemplo, en la adolescencia el desarrollo del auto-concepto involucra el intercambio $y$ retroalimentación de descripciones, creencias $y$ planes con los pares o con las figuras significativas.

Por su parte en la dimensión valorativa o evaluativa, Maggio (2000), sostiene:

La autoestima es el sentimiento valorativo del ser, del conjunto de rasgos corporales, mentales $y$ espirituales que configuran la personalidad (p. 220).

La autoestima es la consideración positiva o negativa del sí mismo. Aparece asociada con la claridad que se tiene del concepto propio $y$ hace referencia específica a la valía personal y esta favorece el sentido de la propia identidad, constituye un marco de referencia desde el que se interpreta la realidad externa y las propias experiencias, influye en el rendimiento, condiciona las expectativas y la motivación y contribuye a la salud y al equilibrio psíquico (Maggio, 2000).

En términos generales la investigación sugiere que la valoración positiva del sí mismo está asociada con la autonomía, seguridad e integridad personales, $y$ que por lo contrario, la valoración negativa se vincula con la ansiedad, el estrés y sentimientos de ineficacia (Abrams \& Hogg, 2003).

Ahora bien, es posible hacer referencia específica a la relación entre la autoestima y la identidad étnica, según Phinney (1990), se ha encontrado una relación directa entre identidad étnica y autoestima, por tanto, este concepto es utilizado como criterio para determinar el impacto de la identidad étnica en el desarrollo psicológico. Uno de los resultados de investigación más interesantes dentro de esta línea de trabajo es la relación positiva entre desarrollo de la identidad étnica y autoestima.

Según la teoría del desarrollo de la identidad étnica planteada por Phinney existen tres momentos importantes o sobresalientes de esta, la primera etapa denominada identidad étnica 
no examinada, se caracteriza por la ausencia de una exploración y toma de decisiones en relación con la etnicidad.

Sigue a esta un período llamado búsqueda de identidad étnica, que se caracteriza por la fuerte exploración de la etnicidad, como resultado de esta búsqueda se da una apreciación más profunda de la propia etnicidad llegando a la etapa de identidad étnica internalizada, es aquí donde los estudios concluyen que las personas expresan mayores niveles de satisfacción personal y autoestima que aquellos que no han trabajado su etnicidad.

En este mismo sentido, se ha observado que la identificación con un grupo étnico discriminado, no lleva necesariamente a un desajuste psicológico, ni es sinónimo de odio hacia los exogrupos, pues los miembros de grupos discriminados no son víctimas pasivas del estigma; por el contrario, en ciertas circunstancias miembros de grupos estigmatizados afrontan las consecuencias negativas del prejuicio con una mayor identificación con su grupo de referencia (Smith, 2001).

\section{MÉTODO}

El presente estudio es de tipo cuantitativo-correlacional, $y$ dentro de las variables exploradas figuró la autoestima, esta se estudió a través del constructo de identidad personal con dos escalas, una sobre auto-concepto (¿cómo soy yo?) y la otra sobre autoestima (¿cómo me valoro?), estas escalas formaban parte de un cuestionario que relacionaba a su vez la identidad personal con otras variables de interés.

\section{PARTICIPANTES}

En el estudio participaron 98 estudiantes de la comunidad de Boruca y 72 estudiantes de la comunidad de Térraba, para un total de 170 personas adolescentes, las edades de esta población joven iba de 12 a 28 años y aunque este representa un rango amplio se consideró importante abarcarlo para conocer si el factor edad determinaba diferencias; aunque el grueso de la población se concentró en edades de entre
12 a 18 años. Todos los participantes, eran estudiantes activos de los colegios de ambas comunidades $y$ algunos de ellos eran estudiantes de escuela.

\section{INSTRUMENTOS}

El instrumento de recolección de información utilizado para este estudio fue el cuestionario auto-aplicado, el cual contenía en la presentación el consentimiento informado, además verbalmente se les indicó que la información solicitada tenía fines de investigación, que sería manejada confidencialmente y que además contaban con el derecho a negarse a participar o bien, dejar el cuestionario en blanco, si así lo consideraban conveniente.

El cuestionario se conformó de tres partes, además de la presentación, una se relacionaba con datos generales, en la cual se exploraron variables sociodemográficas, posteriormente se indagó acerca del auto-concepto y la autoestima.

Para efectos de medir el auto-concepto con ambos grupos se utilizó una lista de adjetivos de manera que los y las participantes eligieron aquellos con los cuales se identificaron $y$ sintieron que les describía de la mejor manera. La escala de respuesta utilizada era de cuatro puntos: 1. Nada, 2. Poco, 3. Bastante y 4. Mucho.

Estos adjetivos contenían criterios positivos y negativos de definición personal (soy una persona atractiva, soy una persona viciosa, soy una persona trabajadora), muchos de ellos ligados con estereotipos atribuidos por la colectividad a las minorías indígenas (Dobles, Fournier y Pérez, 1996). Los Coeficientes Alpha de Cronbach para estas mediciones fueron, para el grupo Boruca de 0,66 y para los Térrabas de 0,70 , es decir, son puntajes moderados, pero aceptables.

Para la medición de la autoestima con ambos grupos se utilizó la escala de autoestima de Rosenberg. Esta escala se compone de 8 ítems, tales como: "Soy una persona valiosa", "Tengo un buen número de cualidades", $y$ se presenta en formato de escala Likert de 4 puntos, donde 1. Muy en desacuerdo y 4. Muy de acuerdo. Cuando fue necesario los ítems 
negativos se re-codificaron, para que el total de las puntuaciones reflejaran altos o bajos niveles de autoestima. Los resultados en esta escala presentaron un Alpha de 0,67 para los Borucas $y$ para los Térrabas 0,60 , puntajes similares a los de la escala anterior y que nuevamente se consideraron aceptables.

\section{PROCEDIMIENTO}

Previo a la aplicación de los cuestionarios se gestionó con el Departamento de Educación Indígena del Ministerio de Educación Pública (MEP) los permisos necesarios para ingresar a las respectivas instituciones educativas y recopilar la información. Seguidamente, se realizó contacto propiamente con las instituciones educativas de interés y sus directores. Además, previamente a la aplicación del cuestionario se llevaron a cabo visitas para efectuar aproximaciones a la comunidad y conocer un poco más de las condiciones de vida de las personas jóvenes y sus familias.

La recolección de datos con el grupo Boruca se realizó en el Liceo de la comunidad de Boruca, en Buenos Aires de Puntarenas, allí fueron aplicados 98 cuestionarios en las aulas, y el tiempo que les tomó completar el cuestionario osciló entre 20 y 30 minutos. La participación fue voluntaria y se aseguró total confidencialidad. El mismo procedimiento se replicó con 72 jóvenes de Térraba con la particularidad de que se incluyó además la población escolar considerada adolescente por superar los doce años de edad.

El trabajo de campo se efectuó entre febrero y noviembre de 2005 posteriormente, los datos fueron procesados $y$ analizados haciendo uso del SPSS v. 10. Una vez que se contó con los resultados, se llevó a cabo una visita a ambas comunidades $y$ formalmente se efectuó una devolución.

\section{RESULTADOS}

DESCRIPCIÓN DE LA MUESTRA

Tanto en Boruca como en Térraba se trabajó con el total de la población de los
Liceos, es decir, se censaron las instituciones, en Boruca participaron 98 estudiantes (43 muchachas y 55 muchachos), y en Térraba 72 personas (38 muchachas y 34 muchachos) estos se auto reconocieron como indígenas, alrededor del $80 \%$ de las personas jóvenes de ambos grupos, sus edades oscilaban entre los 12 y los 18 años.

Con respecto a la distribución por nivel académico, en los Térrabas se tiene que un $11 \%$ de esta población estaba en sexto grado de primaria, $33 \%$ en sétimo año, $38 \%$ en octavo, $y$ un $18 \%$ en noveno. Cabe aclarar que se contó con la participación de estudiantes de primaria, porque muchos de ellos tenían más de 12 años, además es pertinente comentar que en Térraba para ese momento el Liceo sólo tenía disponibilidad para un noveno año, y que en este nivel algunos de los chicos tenían más de 18 años, pero igualmente se incluyeron en el estudio para hacerlos partícipes de la experiencia y porque interesó determinar si el factor edad tenía alguna incidencia de diferenciación entre los jóvenes de menos edad y los mayores.

\section{RESULTADOS DE LA VARIABLE AUTOESTIMA}

En el área de autovaloración o autoestima de jóvenes borucas y térrabas se obtienen resultados positivos, las diferencias entre hombres $y$ mujeres son pequeñas $y$ por lo tanto no se encontraron diferencias estadísticamente significativas con pruebas $t$, pero los promedios indican buenos resultados.

La tabla 1 (página siguiente) contiene datos generales sobre la escala de autoestima por comunidad, en este caso se reportan estadísticas generales y resulta notable la similitud entre puntajes. Ya que para la comunidad de Boruca (98 participantes) la media en autoestima para los jóvenes es de 3,18, mientras que para los residentes en Térraba (72 participantes) este puntaje es de 3,20, en ambos casos la desviación estándar es baja y similar. Lo anterior indica que en ambos grupos se presentan altos niveles de autoestima, en tanto, están muy cerca del 4, el puntaje máximo esperado. 
TABLA 1

PUNTUACIONES GENERALES DE LA ESCALA DE AUTOESTIMA POR COMUNIDAD

\begin{tabular}{lccccc}
\hline & COMUNIDAD & MEDIA & $D E$ & MÍNIMO & MÁXIMO \\
\hline $\begin{array}{l}\text { Escala de } \\
\text { autoestima }\end{array}$ & Borucas & 3,18 & 0,37 & 2,00 & 4,00 \\
\cline { 2 - 6 } & Térrabas & 3,20 & 0,42 & 1,43 & 4,00 \\
\hline
\end{tabular}

Fuente: elaboración propia.

Por otro lado, se revisó la posible interacción de variables demográficas con la autoestima, interesó particularmente el sexo $y$ la edad.

El gráfico 1 presenta los resultados según el sexo, los varones presentan promedios más altos en la escala de autoestima, para los Borucas 3,22 y para los Térrabas 3,21, es decir, puntajes muy cercanos, por otra parte, las mujeres difieren de lo anterior, y son las Térrabas, quienes presentan una media cercana a los puntajes de los varones con 3,19, mientras que las mujeres Borucas puntuaron un 3,12, puntaje que si bien es alto, en tanto se ubica por encima de la media teórica (2), es el más bajo de los cuatro grupos, aspecto que debe ser profundizado en otros estudios considerando posibles factores asociados.

GRÁFICO 1

RESULTADOS DE LA ESCALA DE AUTOESTIMA SEGÚN EL SEXO Y LA COMUNIDAD

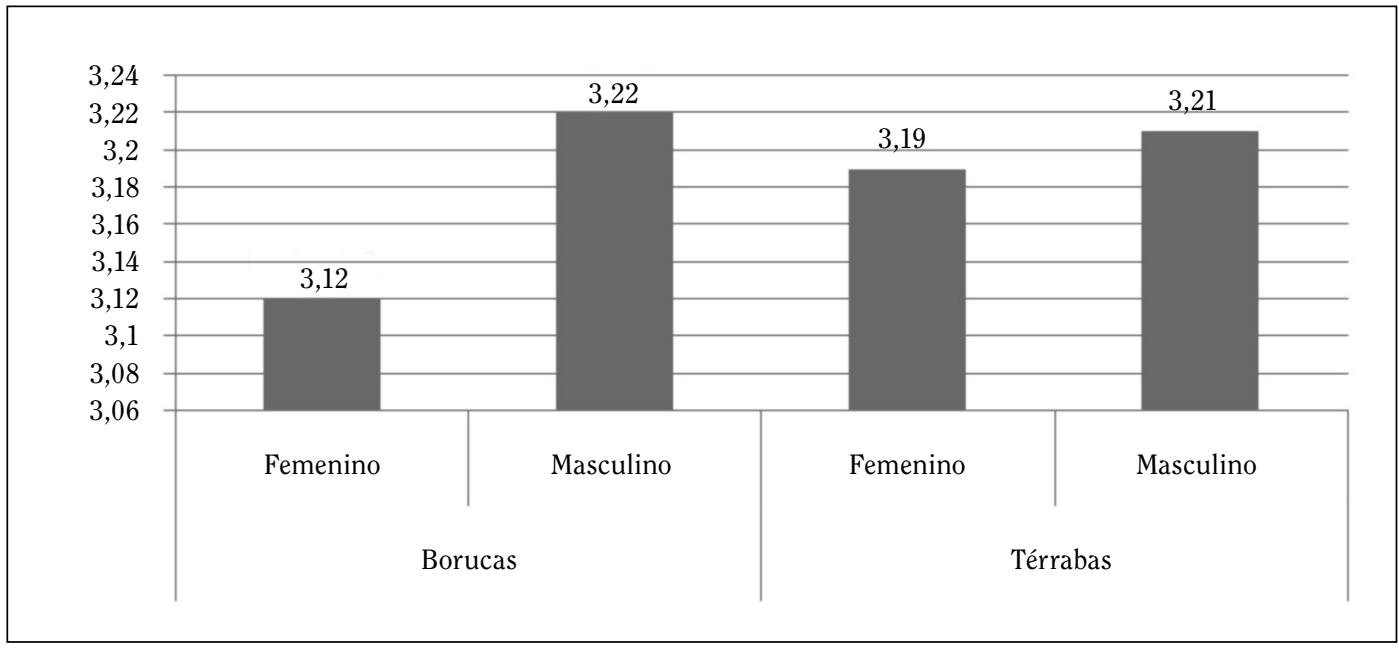

Fuente: elaboración propia.

Otro elemento a tomar en cuenta fue si el factor "edad" influye en la auto-percepción con respecto a la autoestima, como vemos en el gráfico 2 (página siguiente), en ambos grupos hay una leve tendencia a que los jóvenes de mayor edad presentan mayor autoestima que los restantes, aunque las diferencias son pequeñas.
Estos resultados podrían indicar que las personas jóvenes mayores tienen puntajes más altos en autoestima debido a que hay una identidad más consolidada producto de la mayor experiencia, sin embargo estas diferencias sólo fueron significativas en algunos ítems y no son amplias. 
GRÁFICO 2

CRUCE DE VARIABLES SOBRE COMUNIDAD Y EDAD EN LA VARIABLE AUTOESTIMA

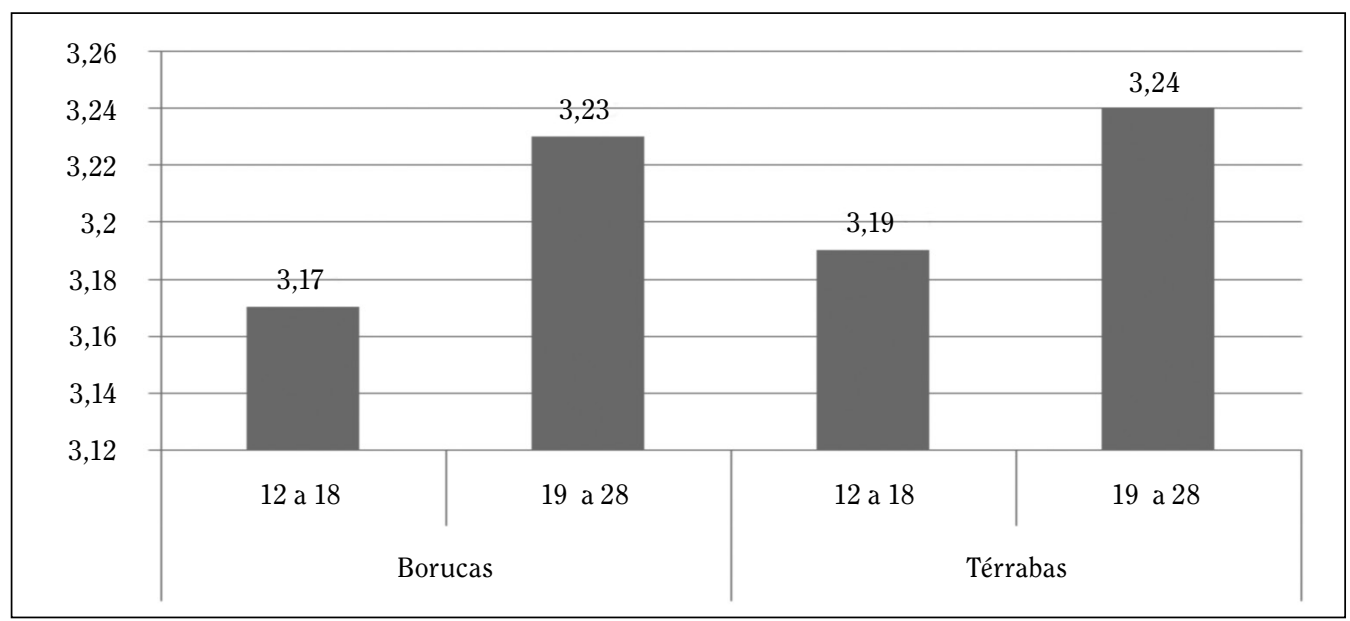

Fuente: elaboración propia.

\section{RESULTADOS DE LA VARIABLE AUTO-CONCEPTO}

Con respecto al auto-concepto se utilizaron una serie de enunciados por medio de los cuales esta población se podía identificar en una escala que iba de 1. Nada a 4. Mucho. Algunos ejemplos de los adjetivos empleados fueron: útil, capaz, atractiva, aburrida, miedosa, vergonzosa entre otros. Así, a mayores puntajes mejor auto-concepto, los ítems con connotaciones negativas fueron invertidos para las sumatorias. Seguidamente, se presentan medidas de tendencia central para revisar este aspecto.
La tabla 2 detalla medidas de interés por comunidad de acuerdo con la agrupación de los adjetivos en positivos $y$ negativos, en este caso se espera entonces que los adjetivos negativos presenten una media menor que los positivos. Ahora bien, los adjetivos positivos para la comunidad de Boruca ascienden a 2,97, mientras que los Térrabas puntúan más alto con 3,15 , por su parte con respecto a los ítems negativos, en los Borucas el puntaje corresponde a 1,88 y para los Térrabas 1,82 . Tal y como se menciona previamente los puntajes de los adjetivos negativos están por debajo de los positivos.

TABLA 2

PUNTUACIONES EN LA ESCALA DE ADJETIVOS POR COMUNIDAD

\begin{tabular}{lcccc}
\hline & \multicolumn{2}{c}{ ADJETIVOS POSITIVOS } & \multicolumn{2}{c}{ ADJETIVOS NEGATIVOS } \\
\cline { 2 - 5 } & BORUCAS & TÉRRABAS & BORUCAS & TERRABAS \\
\hline Media & 2,97 & 3,15 & 1,88 & 1,82 \\
Mediana & 3,00 & 3,12 & 1,87 & 1,75 \\
Moda & $2,63(\mathrm{a})$ & 3,38 & 2,00 & 1,75 \\
Desviación estándar & 0,41 & 0,45 & 0,42 & 0,46 \\
Mínimo & 2,00 & 1,88 & 1,13 & 3,00 \\
Máximo & 4,00 & 4,00 & 3,13 & 3,00 \\
\hline Número de participantes & 98 & 72 & 98 & 69 \\
\hline
\end{tabular}

Fuente: elaboración propia.

a. Existen varias modas. Se muestra el menor valor. 
Para profundizar estas diferencias y determinar si existían diferencias significativas entre medias por comunidad, se efectuó una prueba $t$ para muestras apareadas, los datos resultantes se encuentran en la tabla 3 . Y los resultados del análisis indican que para ambas comunidades existe una diferencia significativa entre las medias comparadas, es decir, la población joven de las dos comunidades identifican en sí mismos mayor nivel de adjetivos positivos que negativos.

Se observa además una leve tendencia a que los jóvenes térrabas puntúan más alto en los adjetivos positivos y más bajo por su parte en los negativos, factor que sería interesante de explorar posteriormente.
Estos datos son valiosos en tanto, permiten cuestionar algunos planteamientos que asocian la baja autoestima con ciertos grupos sociales, tales como las personas pertenecientes a etnias indígenas y las personas adolescentes.

Finalmente, interesó efectuar un análisis de correlación de Pearson entre las escalas estudiadas, a saber, autoestima y los adjetivos positivos y negativos. Los resultados de esta medición se encuentran en la tabla 4 y según los mismos, se halla una correlación significativa y moderada entre la escala de autoestima y los adjetivos positivos, es decir, las personas jóvenes de ambas comunidades $(r=0,48, r=0,48)$, entre mayor autoestima puntuaban tendían a identificar más aspectos descriptivos positivos en sí mismos.

TABLA 3

PRUEBA T PARA MUESTRAS APAREADAS SEGÚN ADJETIVOS POSITIVOS Y NEGATIVOS POR COMUNIDAD

\begin{tabular}{|c|c|c|c|c|c|c|}
\hline & & MEDIA & $\mathrm{DE}$ & $\mathrm{T}$ & GL & SIG \\
\hline \multirow{2}{*}{ Borucas } & Positivos & 2,97 & 0,41 & \multirow{2}{*}{17,60} & \multirow{2}{*}{97} & \multirow{2}{*}{0,00} \\
\hline & Negativos & 1,88 & 0,42 & & & \\
\hline \multirow{2}{*}{ Térrabas } & Positivos & 3,13 & 0,44 & \multirow{2}{*}{15,38} & \multirow{2}{*}{68} & \multirow{2}{*}{0,00} \\
\hline & Negativos & 1,82 & 0,46 & & & \\
\hline
\end{tabular}

Fuente: elaboración propia.

RELACIÓN ENTRE LA AUTOESTIMA

Y EL AUTOCONCEPTO

Lo mismo sucede con la autoestima y los adjetivos negativos, con una relación inversa, es decir, entre mayor autoestima o valoración personal, menor asignación de adjetivos negativos hacia sí mismos, en este caso con correlaciones de menor tamaño pero igualmente significativas $(r=-0,32, r=-0,32)$.

TABLA 4

MATRIZ DE CORRELACIONES ENTRE LAS ESCALAS DEL ESTUDIO

\begin{tabular}{cccc}
\hline \multirow{2}{*}{ Borucas } & & ADJ POSITIVOS & ADJ NEGATIVOS \\
\cline { 2 - 4 } & Autoestima & $0,49^{* *}$ & $-0,32^{* *}$ \\
\cline { 2 - 4 } & Adj. Positivos & - & $-0,06$ \\
\hline \multirow{2}{*}{ Térrabas } & Autoestima & $0,48^{* *}$ & $-0,32^{* *}$ \\
\cline { 2 - 4 } & Adj. Positivos & - & $-0,20$ \\
\hline
\end{tabular}

Fuente: elaboración propia. $\quad$ ** La correlación es significativa al nivel 0,01 
Por último, como se espera los adjetivos no poseen correlaciones significativas entre sí.

\section{CONCLUSIONES Y DISCUSIÓN}

Según Tajfel (1984) es una característica humana bastante general el tratar de lograr y conservar la estima propia y la de los demás, para la mayoría de las personas es importante entonces tener $y$ mantener una autoimagen positiva, y según este autor, el tener que vivir con una imagen despreciable de uno mismo, tanto si procede de dentro como de los demás, constituye un grave problema psicológico.

En este estudio se exploró el autoconcepto y la autovaloración en jóvenes pertenecientes a dos grupos indígenas, en un momento clave del desarrollo como lo es la adolescencia, donde las comparaciones entre personas y grupos constituyen mecanismos recurrentes para la conformación de la identidad.

Ambas comunidades indígenas (Borucas y Térrabas), han perdido parte importante de su acervo cultural, lo que puede resultar en una identificación menos intensa por parte de la juventud con su etnia indígena en comparación con otros grupos, sin embargo, estos jóvenes reflejaron tener un importante sentido de pertenencia hacia su grupo étnico.

Los resultados señalan que la identidad personal de jóvenes borucas y térrabas reflejó resultados positivos, es decir, tanto el auto concepto como la valoración personal son en este grupo satisfactorios, sin embargo, en el estudio más amplio se evidenció que la valoración $y$ visión de sí mismos es independiente de la identificación que tenía esta población adolescente con su cultura indígena o con su cultura dominante.

Esto llama la atención pues se ha constatado que la autoestima en grupos étnicos minoritarios, está fuertemente ligada con la etnicidad y con la relación con los grupos dominantes. Sin embargo, cabe reiterar que por las particularidades de cercanía y contacto con personas no indígenas que tienen estas poblaciones, las aristas que han surgido a raíz de la aculturación y la propia vivencia de la bicultu- ralidad, sean difíciles de precisar, de manera que su identidad personal no esté tan determinada por su pertenencia o no al grupo indígena.

Respecto de los datos sociodemográficos en los grupos estudiados, se determinó que el sexo y la edad contribuyen a encontrar pequeñas diferencias, pero que no son estadísticamente significativas, lo cual indica que los criterios en cuanto a autoimagen y valoración se comportan de forma similar para hombres $y$ mujeres.

Las personas jóvenes de ambas comunidades reportan datos que se asocian con salud emocional y una fuerte autoestima, es decir, no presentan mayores diferencias al respecto, lo cual indica que las estructuras geográficas, económicas y sociales tienden a constituir espacios de desarrollo socio personal similares.

Los procesos de construcción de identidad se saben complejos, $y$ más aún en el caso de identidades que comprenden lo étnico. Sus auto conceptos no sólo reflejan una conciencia históricamente compartida que los inspira a involucrarse con sus identidades indígenas, sino que ellas también reflejan sus experiencias sociales tanto de sus comunidades nativas como de las sociedades dominantes.

Según Smith en sociedades pluri-culturales como la nuestra, estratificadas por clase social, género, etnia y nacionalidad, hay que tener en cuenta que el significado particular y el impacto psicológico de pertenecer a determinado grupo sociocultural adquiere significado sólo en relación con otros colectivos (Smith, 2002b).

Así, según Tafjel (1984) las comparaciones valorativas con otros grupos o con miembros individuales de los mismos pueden convertirse en aspectos importantes de la autoimagen de una persona, especialmente cuando se pertenece a una minoría que se considera claramente marginada de los otros y (explícita o implícitamente) inferior a ellos en aspectos importantes.

En este estudio el punto de partida fue el interés por explorar la identidad en función de aspectos socialmente reconocidos como los adjetivos y descripciones hacia sí mismos, así como las valoraciones que se tienen. Condición que si previamente es importante, adquiere 
mayor relevancia entre el grupo estudiado donde hay un proceso intenso por la definición del sí mismo, como también un bombardeo constante reforzado hoy por la tecnología para delimitar la identidad de los jóvenes a espacios predeterminados $y$ homogenizados.

Según Rosenberg (1965) el proceso de autoestima en la adolescencia es especialmente crucial porque es el momento en el que las personas tienen que lidiar más con cambios significativos en los niveles físico y sexual, con un rápido desarrollo mental, psicosocial y moral, $y$ estos cambios también hacen que se reevalúen $y$ se redefinan constantemente.

Particularmente en estas comunidades se pudo observar cómo confluyen elementos en el significado de auto concebirse indígena con el ser adolescente, vivir en zona rural y tener proyecciones $y$ limitaciones específicas para el futuro, por sus condiciones socio demográficas.

Los altos puntajes en identidad personal sugieren que puede haber una serie de factores que podrían ser protectores respecto a la constitución de una autoestima positiva en la población joven de estas comunidades (aunque no haya habido una relación directa con su identidad étnica), sí se puede suponer que el hecho de que las y los jóvenes hayan crecido y permanezcan en sus territorios indígenas puede contribuir a una identidad personal positiva e incluso a un mejor rendimiento académico, por el soporte que pueden suministrar las redes sociales, ya que como se evidenció en otros estudios (Delgado, 2007) cuando los jóvenes se percibían como indígenas pero no habían tenido la experiencia de vivir en reservas, tendían a presentar puntuaciones más bajas en autoestima.

Otro elemento facilitador de una autoestima positiva podría ser el hecho de que en las comunidades estudiadas existen mayorías de jóvenes indígenas en los espacios de interacción social como la escuela y el colegio, etc. Por tanto, como lo señaló un informante clave, en estos espacios se da un fenómeno de inversión de roles donde el grupo históricamente marginado (las y los indígenas) resulta ser el grupo dominante $y$ los chicos $y$ chicas no indígenas resultan ser las personas objeto de discriminación.
Queda pendiente entre otras interrogantes, indagar que sucede al pasar del tiempo con la identidad de los jóvenes indígenas de estas comunidades, si hay una tendencia a la estabilidad y consolidación en los patrones de autoestima y autoconcepto, y ¿qué sucederá cuando estos muchachos y muchachas trascienden los espacios de sus comunidades e ingresen al mundo adulto?, que por las características socioeconómicas de estas comunidades, normalmente va a deparar el enfrentamiento más directo $y$ constante a relacionarse con las personas no indígenas e incluso, a salir de sus territorios.

\section{FUENTES}

Abrams, D. y Hogg, M. Collective Identity: Group Membership and Self Conception. Blackwell Handbook of Social Psychology: Group Processes. 18. 2003: 425-460.

Bozzoli, M.; Guevara, M. Los indigenas costarricenses en el siglo XXI. Ideario Costarricense. San José, CR: Editorial UNED, 2002.

Delgado, E. The Construction and Experience of Indigenous Nations Identity: Implications for Well-Being And Academic Persistence. University of Kansas. Obtenido el 13 de Marzo del 2008 de la base de datos EBSCOHost Academic Search Elite. July, 2007.

Dobles, I.; Fournier, M. y Pérez, R. "Representaciones sociales del quinto centenario del arribo de los Españoles a América y su relación con la identidad social”. Pérez, R.; Dobles, I. y Cordero, T. (Eds.). Psicología social: Dominación social y subjetividad. San José, CR. Editorial de la Universidad de Costa Rica, 1996: 57-92.

Engler, B. Introducción a las teorías de la personalidad. México: McGraw-Hill, 1998. 
Maggio,E. Aportes de la clínica al conocimiento del individuo. McGraw-Hill, 2000.

Nájera, P. Recopilación de historias Térrabas. Rincón Ecológico Térraba, 2004.

Phinney, J. "Ethnic identity in Adolescents and Adults: Review of Research". Psychological Bulletin 3. 1990: 499-514.

Phinney, J. "Ethnic identity and self-esteem: A Review and integration". Hispanic Psychology 13. 1995: 193-208.

Ricciardelli, L.; McCabe, M.; Ball, K. y Mellor, D. "Sociocultural Influences on Body Image Concerns and Body Change Strategies Among Indigenous and Non-Indigenous Australian Adolescent Girls and Boys". Sex Roles 51, 11/12. Obtenido el 12 de febrero del 2008 de la base de datos Proquest. December, 2004.

Rojas, D. "El conflicto entre tradición y modernidad: Construcción de la identidad cultural indígena Bribrî". Cuaderno de Ciencias Sociales. San José: Facultad Latinoamericana de Ciencias Sociales, 2002.
Rosenberg, M. Society and the adolescent self image. NJ: Princeton University, 1965.

Smith, V. "Aportes a la comprensión de la identidad étnica en niños y niñas de grupos étnicos minoritarios". Revista de Ciencias Sociales 98. 2002a: 71-82.

Smith, V. "Relaciones Interétnicas en Costa Rica: Redacción de 3 artículos sobre actitudes interétnicas e identidad étnica en jóvenes mestizos y afrocostarricenses". Informe de Investigación. Instituto de Investigaciones Psicológicas, Instituto de Investigaciones Sociales. Universidad de Costa Rica. 2002b.

Smith, V. "Discriminación percibida $y$ autoestima en jóvenes de grupos minoritarios y mayoritarios en Costa Rica”. Revista Interamericana de Psicología 39 (1). 2005.

Tajfel, H. y Turner, J. "An integrative theory of intergroup conflict". Worchel, S. y Austin, W. G. (Eds.). The social psychology of intergroup relations. Monterey, CA. Brooks/Cole, 1979: 33-47.

Tajfel, H. Grupos humanos y categorías sociales. Barcelona: Editorial Herder, 1984. 